\title{
Develop a Mobile Nursing App to Improve Nursing Workflow and Effectiveness Assessment
}

\author{
Chin-Yuan TSAN ${ }^{\mathrm{a}}$, Meng-Chun CHEN ${ }^{\mathrm{b}}$, Jia-Chang $\mathrm{WEN}^{\mathrm{c}}$ and Yi-Chen WANG ${ }^{\mathrm{d}}$ \\ ${ }^{a}$ Nursing Supervisor, National Taiwan University Hospital / Nurse Director, \\ National Taiwan University Hospital, Yunlin Branch, Taiwan \\ ${ }^{\mathrm{b}}$ Nursing Information, National Taiwan University Hospital, Taiwan \\ ${ }^{\mathrm{c}}$ Information Manager, National Taiwan University Hospital, Taiwan \\ ${ }^{\mathrm{d}}$ Nursing Information, National Taiwan University Hospital, Taiwan
}

\begin{abstract}
We develop a mobile care application that includes tools such as voice input, image upload, and image recognition. This procedure will be used in clinical care. The study is expected to undergo actual use testing in the ward and a questionnaire survey three months after use. During use, the mobile phone connection data will continuously monitor to analyze the number and time of connection records.
\end{abstract}

Keywords. Mobile nursing APP, speech recognition, image recognition

\section{Introduction}

The existing nursing station computer and mobile cart are flexible in terms of volume or movement. It is not enough to support the nurses to find the laptop to record when they need it. At present, many medical centers in Taiwan have begun to adopt nursing information systems, but they still cannot reduce the burden on nursing staff when carrying out various nursing work. Therefore, we have developed a mobile Nursing APP so that the nurse can quickly perform clinical records in the light-loaded state, making up for the dead spots of the electronic document.

\section{Methods}

The most significant weakness of the mobile phone relative to the $\mathrm{PC}$ is the inconvenient keyboard typing. Therefore, we introduced speech recognition input instead of keyboard text input. At present, our speech recognition success rate has exceeded 95\%; using the camera lens equipped on the mobile device; the photos are store in the electronic records; using Azure image text recognition technology, the text content identification tool is applied to the chemotherapeutic injection drug review, all data are compared and confirmed before passing on to the next step. The images generated by the related image recognition and the results, including the identification, are stored at the moment. This study is expected to carry out actual use tests and conduct a questionnaire survey of the 
unit's nursing staff three months after use. During the use of each ward, the mobile phone connection data is continuously monitored for three months, and connection records have been performed. Use frequency and use time analysis as a benchmark for subsequent expansion of the whole hospital.

\section{Results}

The mobile nursing app system was combined with the unique executable on the mobile phone, including speech recognition, image uploading, and image recognition. For example, using speech recognition to identify the result of vital signs, using camera to taking picture of the wound and store in nursing record to observe the changes of the wound, use image text recognition tools to review the settings of the instrument and assist in the identification of patients and drugs to ensure correct medication administration. At present, the system has been used for three months in one unit, and there is 22 nursing staff in the word. The average value of the questionnaire is 3.59. At present, it appears that the nursing staff is tending to be satisfied with the use of the nursing APP; the analysis of connection data shows that the monthly usage has shown a steady increase of about $15 \%$, and the most frequently used item is shift notes.

\section{Conclusions}

Design mobile applications' primary purpose is to help users streamline their work to be done quickly, compared to the data query, data entry feature to provide the actual enhance operational efficiency, and features of this system is a combination of digital technology in nursing practice on clinical nursing. The results show that nurses can improve work continuity and efficiency through alternate use. We suggest that when determining the implementation of nursing APP systems, the functions that are related to workflow with the ability to increase performance should be considered first.

\section{Acknowledgements}

This study can be successful, thanks to the head of the nursing department of the National Taiwan University Hospital and the help of the Information department.

\section{References}

[1] Bautista JR. (2019). Filipino nurses' use of smartphones in clinical settings. Computers Informatics Nursing, 37(2), 80-89. doi: 10.1097/CIN.0000000000000482

[2] Choi M, Lee H, Park JH. (2018). Effects of using mobile device-based academic electronic medical records for clinical practicum by undergraduate nursing students: A quasi-experimental study. Nurse Education Today, 61, 112-119. doi: 10.1016/j.nedt.2017.11.018

[3] Choi M, Lee HS, Park JH. (2015). Usability of academic electronic medical record application for nursing students' clinical practicum. Healthcare Informatics Research, 21(3), 191-195. doi: 10.4258/hir.2015.21.3.191 
[4] Liu CF, Cheng TJ. (2015). Exploring critical factors influencing physicians' acceptance of mobile electronic medical records based on the dual-factor model: A validation in Taiwan. BMC Medical Informatics And Decision Making, 15, 4. doi: 10.1186/s12911-014-0125-3

[5] Scher D L, Jarrin R, Chan J, Rosen H. (2013). mHIMSS Roadmap Mobile Health Apps: A practical guide for healthcare stakeholders.

[6] Tahamtan I, Pajouhanfar S, Sedghi S, Azad M, Roudbari M. (2017). Factors affecting smartphone adoption for accessing information in medical settings. Health Information and Libraries Journal, 34(2), 134-145. doi: 10.1111/hir.12174 